\title{
IMMEDIATE AND LONG-TERM EVOLUTION ASSESSMENT AFTER SURGERY ON THE SAPHENOUS VEIN WITH THE MODIFIED CHIVA TECHNIQUE
}

\author{
Marta Osório de Moraes ${ }^{1}$, Eliane Yumi Fuji ${ }^{1}$, Francisco Winter dos Santos Figueiredo ${ }^{2}$, \\ Jonathan Souza Sarraf ${ }^{2,3,4}$, Taynah Cascaes Puty ${ }^{2,3}$, Wallingson Michael', \\ Fernando Adami ${ }^{2,3}$, João Antônio Correa ${ }^{1}$ \\ ${ }^{1}$ Clinic research department, ABC School Medicine, Santo Andre, SP, Brazil \\ ${ }^{2}$ Faculdade de Medicina do ABC, Laboratório de Epidemiologia e Análise de dados, \\ Santo André- São Paulo, Brazil \\ ${ }^{3}$ Oncológica do Brasil - Ensino e Pesquisa \\ ${ }^{4}$ Post Graduate in Genetic and Molecular Biology - Federal University of Pará
}

\section{ABSTRACT}

INTRODUCTION: The preservation of the great saphenous vein (GSV) has increasingly been considered when surgery for the treatment of varicose veins is indicated when complications from degenerative diseases, such as atherosclerosis at a coronary level and peripheral venous insufficiency occur. Thus, the autologous GSV is considered the best substitute for arterial revascularization. The technique that preserves the great saphenous vein is the Conservative Ambulatory Hemodynamic Management of Varicose Veins (CHIVA), which is widely used. Considering the importance and the use of this technique, the objective of this study was to evaluate the immediate and long-term evolution after surgery on the saphenous vein with the modified CHIVA technique.

MATERIALS AND METHODS: A prospective observational study with data from individuals who underwent surgery for the preservation of the GSV by the modified CHIVA technique with the removal of varicose tributaries without ligation of the saphenofemoral junction. Data collection was performed from March 2014 to January 2015 at Padre Anchieta Hospital in Sao Bernardo do Campo, São Paulo. The variables were the great saphenous vein patency assessed by means of vein diameter, as described above, and the presence or not of reflux during the preoperative assessment, on the 30th day after surgery, and at the sixth month after surgery.

RESULTS: The main results showed that there were no statistically significant differences in the saphenous vein diameter in any of the evaluated segments and in regard to the presence of reflux when comparing the pre-operative period to 30 days after CHIVA and to six months after CHIVA.

Address for correspondence:

Marta Osório de Moraes

Laboratório de Epidemiologia e Análise de Dados

Faculdade de Medicina do ABC

821. Príncipe de Gales Ave

Santo André, SP CEP 09060-650

Brasil

e-mail:martaosoriomoraes@hotmail.com

Received: December 20, 2017

Accepted: January 30, 2018
CONCLUSION: This study did not show change in the diameter of the veins, nor recurrence of reflux in the lower limbs, thus, it showed good results in the short, medium, and long term. There was no relationship between the diameter reductions of veins during the follow-up period.

Keywords: CHIVA technique, saphenous vein, long term 


\section{INTRODUCTION}

The life expectancy of the general population has shown an increase in the last decades, and, in the same proportion, an increase in the number of complications from degenerative diseases, such as atherosclerosis at coronary level and peripheral venous insufficiency, that occur $(1,2)$. In these cases, preservation of the great saphenous vein (GSV) has increasingly been considered when surgery for the treatment of varicose veins is indicated $(1,3)$.

Most of the times, the changes that affect varicose veins are caused by reflux in the saphenofemoral junction (SFJ) due to ostial valvular insufficiency, extending to their trunk and often being transferred to their collateral superficial veins $(4,5)$. Sometimes, the mitral SFJ is competent and the changes of the saphenous vein caused by reflux might linked to other sources, such as insufficient perforating veins that connect to it, especially the Hunter's perforator, which is situated in the middle third of the thigh, or transfer of reflux through the collateral veins that connect the GSV to the external saphenous vein due to insufficiency of the saphenopopliteal junction (SPJ) $(6,7)$.

Thus, the autologous GSV is considered the best substitute for arterial revascularization of the lower limbs and it can be used in surgeries $(3,8)$. In the clinical practice of the field of varicose vein surgeries, preservation of the GSV is considered when it is normal on clinical examination or when there is discreet amendment in continuous-wave Doppler ultrasonography $(6,9)$. In addition, non-stripping of the GSV can reduce injuries of the lymphatic vessels and saphenous nerve that accompany it, resulting in a lower risk of lymphatic and nerve alterations in the postoperative period of varicose vein surgery (1).

The main surgical techniques for the preservation of the great saphenous vein are ligation of the SFJ, ligation and sectioning of the junction, ligation of aortic arch tributaries, and many others $(10,11)$. However, the technique that preserves the great saphenous vein is the Conservative Ambulatory Haemodynamic Management of Varicose Veins (CHIVA), which is widely used. This technique consists of ligation of the internal saphenous vein with reflux at the level of the femoral vein with a staged resection of tributaries to maintain the reentry of blood into the deep venous system prevents occlusion of the tribu- taries of the aortic arch and insufficiency of the perforating veins that connect to the GSV $(9,12)$.

Nevertheless, despite the importance and the use of this technique, there is still a lack of followup studies on individuals who were subjected to the CHIVA technique for different periods of time. The objective of this study was to evaluate the immediate and long-term evolution after surgery on the saphenous vein with the modified CHIVA technique.

\section{MATERIALS AND METHODS}

A prospective observational study with data on individuals who underwent surgery for the preservation of the great saphenous vein by the modified CHIVA technique with the removal of varicose tributaries without ligation of SFJ.

Data collection was performed from March 2014 to January 2015 at Padre Anchieta Teaching Hospital in Sao Bernardo do Campo, São Paulo, with the approval of the Research Ethics Committee of the Faculdade de Medicina do ABC (No 00695512.8.1001.0082).

The individuals included over 18 years of age and presented with primary trunk varicose veins, presence of reflux in the saphenous vein with dilation (with diameter smaller than $10 \mathrm{~mm}$ ), and good clinical conditions. Those who had chronic deep vein insufficiency (saphenopopliteal junction of the external saphenous vein), or those who presented active ulcers, or arterial lymphatic disease or were pregnant were excluded.

The individuals were evaluated with color Doppler in the preoperative period, on the $30^{\text {th }}$ day after surgery, and at the sixth month after surgery, always by the same evaluator. Ultrasonographic diagnostics was performed with the diagnostic Ultrasound T3000 with a high frequency linear transducer of 5 to $12 \mathrm{MHz}$.

Individuals in orthostasis were examined and the reflux was assessed using the Valsalva maneuver and distal compression of the limbs. B mode (bidimensional image) was utilized for the study of the vein anatomy and for compressibility, ruling out thrombophlebitis or deep venous thrombosis. In addition, color mode (color mapping) and pulsed mode were utilized to complement the study of vessels and the analysis of the presence of valve insufficiency. 
For performing the Doppler, five topographic points were defined for the measurement of the internal saphenous vein in the lower limb: (i) SFJ; (ii) proximal thigh (10 $\mathrm{cm}$ below the SFJ); (iii) distal thigh (10 $\mathrm{cm}$ above the knee); (iv) proximal leg (10 $\mathrm{cm}$ below the knee); (v) distal leg (10 $\mathrm{cm}$ above the medial malleolus).

The variables studied were the great saphenous vein patency assessed by means of vein diameter, as described above, and the presence or not of reflux during the preoperative assessment, on the $30^{\text {th }}$ day after surgery, and at the sixth month after surgery.

\section{RESULTS}

Twelve individuals who underwent surgical treatment of varicose veins with the modified CHIVA technique with staged resection of varicose veins without ligation of the SFJ were studied.

Table 1 shows the median diameter $(\mathrm{cm})$ of the segments evaluated in the preoperative period, on the $30^{\text {th }}$ day after surgery, and at the sixth month after surgery. However, there were no statistically significant differences $(p>0.05)$ in the diameter changes of the saphenous vein segments in the different observed periods in both lower limbs.

Table 1. Median diameter $(\mathrm{cm})$ of the great saphenous vein in the evaluated regions in the preoperative period, on the $30^{\text {th }}$ day after surgery, and at the sixth month after surgery in the right lower limb and the left lower limb

\begin{tabular}{|c|c|c|c|c|}
\hline \multicolumn{5}{|c|}{ Right lower limb } \\
\hline \multirow{3}{*}{ Region } & & & & \multirow{3}{*}{$\mathrm{p}^{*}$} \\
\hline & Preoperative & $30^{\text {th }}$ day & $6^{\text {th }}$ month & \\
\hline & \multicolumn{3}{|c|}{ Median diameter $(\mathrm{cm})$} & \\
\hline \multicolumn{2}{|l|}{ Saphenofemoral junction } & \multicolumn{2}{|c|}{0.888} & 0.645 \\
\hline \multicolumn{2}{|l|}{ Thigh (proximal) } & 0.430 & 0.500 & 0.688 \\
\hline \multicolumn{2}{|l|}{ Thigh (distal) } & 0.460 & 0.540 & 0.470 \\
\hline \multicolumn{2}{|l|}{ Leg (proximal) } & 0.420 & 0.485 & 0.780 \\
\hline Leg (distal) & 0.410 & 0.380 & 0.335 & \multirow[t]{2}{*}{0.311} \\
\hline \multicolumn{4}{|c|}{ Left lower limb } & \\
\hline \multirow{3}{*}{ Region } & \multicolumn{3}{|c|}{ Period } & \multirow{3}{*}{$\mathrm{p}^{*}$} \\
\hline & Preoperative & $30^{\text {th }}$ day & $6^{\text {th }}$ month & \\
\hline & \multicolumn{3}{|c|}{ Median diameter $(\mathrm{cm})$} & \\
\hline Saphenofemoral junction & 0.970 & 0.980 & 0.910 & 0.487 \\
\hline Thigh (proximal) & 0.600 & 0.643 & 0.625 & 0.914 \\
\hline Thigh (distal) & 0.510 & 0.503 & 0.445 & 0.895 \\
\hline Leg (proximal) & 0.475 & 0.448 & 0.485 & 0.760 \\
\hline Leg (distal) & 0.410 & 0.310 & 0.295 & 0.181 \\
\hline
\end{tabular}

${ }^{\star}$ Kruskal-Wallis test

Qualitative variables were presented by relative frequencies and analyzed by the chi-square test, while the quantitative analyses were presented by medians and analyzed by the Kruskal-Wallis test through the non-normal data (Shapiro Wilk test, $\mathrm{p}<0.05$ ). The adopted significance level was $95 \%$. The program used was the Stata version 11.0.
In regard to the presence of reflux in the evaluated segments in the preoperative period, on the 30th day after surgery, and at the sixth month after surgery, Fig. 1 shows that both in the right lower limb (Fig. 1A) and the left lower limb (Fig. 1B) there were no statistically significant differences for any of the periods $(\mathrm{p}=0.346 ; \mathrm{p}=0.456$ and $\mathrm{p}=0.111$, respectively in the right, and $\mathrm{p}=0.408, \mathrm{p}=0.582$ and $\mathrm{p}=0.848$, in the left). 

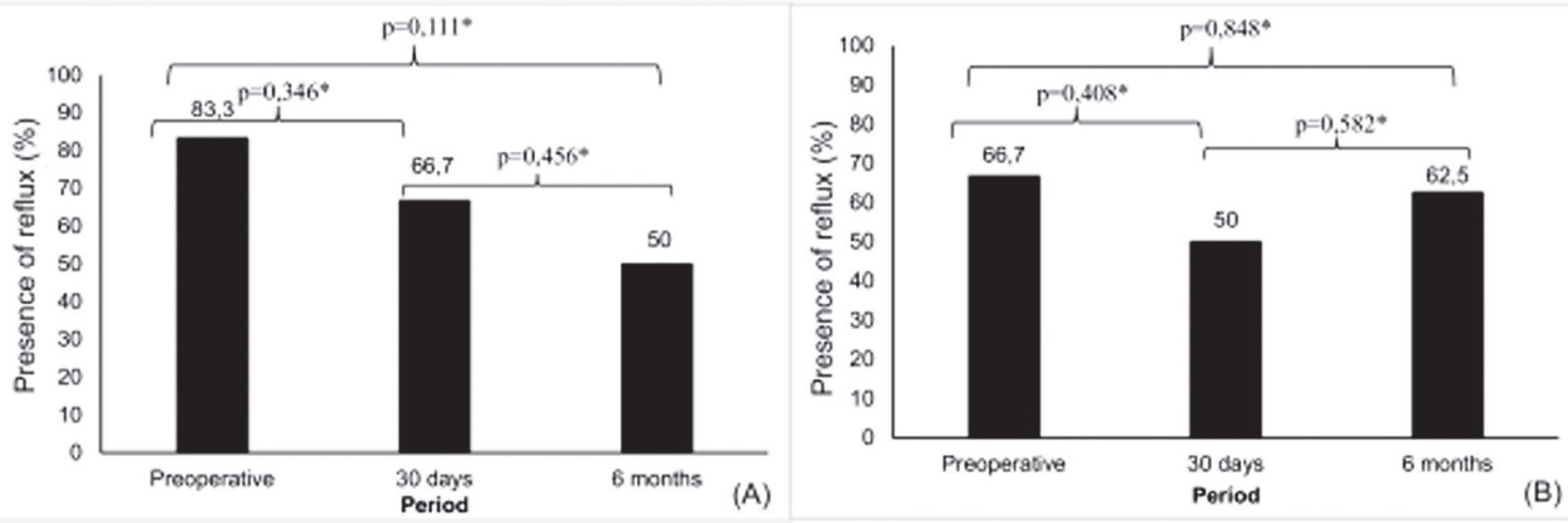

Fig. 1. Presence of reflux (\%) in the preoperative period, on the 30th day after surgery, and at the sixth month after surgery in the right lower limb (A) and the left lower limb (B)

\section{DISCUSSION}

There are many studies on the treatment of chronic venous disease, but trials comparing the conventional procedure and the CHIVA technique in the treatment of chronic venous insufficiency are scarce. Although this technique has been used for more than ten years, little is known about the outcomes in the individuals who were subjected to it. The main results stated that there are no statistically significant differences in the saphenous vein diameter in any of the evaluated segments and in the presence of reflux when comparing the pre-operative period to 30 days after CHIVA and to six months after CHIVA.

Studies about the preservation of the saphenous vein with a technique similar to that observed in this study are scarce, mainly with respect to the time of monitoring of medium and long-term results in individuals, such as the ones in this study, thirty days and six months after CHIVA, respectively. Thus, it is possible to evaluate individuals by longitudinal research, verifying not just the state of the saphenous veins, and not only short term (30 days), but also medium term (6 months).

This study did not show statistically significant differences in the reduction in the GSV diameter since all veins remained patent, without signs of occlusion, and it was possible to use them as arterial substitute. There is a chance of failure when using the CHIVA technique (13) due to vessel occlusion but such was not observed in this study. Other studies have supported the effectiveness of this therapy in venous reflux, studying leg ulceration (14).
Carandina et al. (15) found less reflux regress in group treated with CHIVA when compared to stripping in 10 years of follow-up, showing the efficacy of this treatment in the long-term as well. Moreover, the presence of reflux observed in this study after the technique should not be considered as failure of treatment because it occurs due to the drainage of the region in accordance with physiological hierarchy promoted by CHIVA (13).

In the long-term monitoring, the literature shows a favorable outcome for CHIVA regarding the recurrence of the symptoms due to obstruction after surgery, when evaluated by experienced professionals (15), clinical examination, quality of life scale, and venous Doppler of superficial and deep venous systems of the lower limbs. Other studies (16) performed in different periods of this study also could not find statistical difference in the reduction of the GSV diameter compared to the results of the CHIVA. Despite the small sample, our data are similar to other studies already done and does not show any correlation between the saphenous vein diameter in relation to the results with the CHIVA technique.

When Rollo et al. (16) used a technique resembling that observed in this study, they found that the lower saphenous vein was preserved in its entire length in $90 \%$ of cases, and its average diameter decreased significantly compared to the preoperative period, but continued to be suitable for use as arterial substitute.

The reduced number of individuals and the follow-up time, which, although being longer than most of the presented studies, it still seems to be short to 
Marta Osório de Moraes, Eliane Yumi Fuji, Francisco Winter dos Santos Figueiredo et al.

evaluate the obstruction of saphenous vein can be highlighted as the limitations of this study. For data improvement, rigorous technical criteria were implemented for assessing the evaluated factors. The control with Doppler used for assessment gave us data reliability as it is currently regarded as the test of choice for the evaluation of the superficial and deep venous system, and because it is able to locate valvular incompetence with high sensitivity and specificity when compared to other methods, such as phlebography and plethysmography $(17,18)$.

In order to reduce information bias and interference with the results observed in the GSV, all individuals were evaluated by the same examiner, following the same protocol, and cases of reflux in the deep venous system and external saphenous vein were excluded because there is an important association between both $(10,13)$.

\section{CONCLUSION}

The CHIVA technique besides being considered secure for treatment of varicose veins, is widely used in the research world $(15,19)$. This study did not manage to find a change in the diameter of the veins, nor recurrence of reflux in the lower limbs. Further studies containing larger sample of patients are necessary, as well as the increase in postoperative follow-up time, so that the real correlation between these evaluated factors and the evolution of individuals who underwent surgery with the CHIVA technique can be analyzed.

\section{REFERENCES}

1. Franceschi C. Pour une cure conservatrice et hemodynamique de l'insufficience veineuse en ambulatoire: CHIVA. Angiologie. 1988;72:99-100.

2. Maffei F, HA R. Trombose venosa profunda dos membros inferiores: incidência, patologia, fisiopatologia e diagnóstico. Doenças vasculares periféricas. 2002;3:1363-86.

3. Goldman S, Zadina K, Moritz T, Ovitt T, Sethi G, Copeland JG, et al. Long-term patency of saphenous vein and left internal mammary artery grafts after coronary artery bypass surgery: results from a Department of Veterans Affairs Cooperative Study. J Am Coll Cardiol. 2004;44(11):2149-56. doi: 10.1016/j.jacc.2004.08.064

4. Giannini M, Rollo HA, Maffei FHdA. Role of duplex scanning in the diagnosis of asymptomat- ic lower-extremity deep vein thrombosis. Jornal Vascular Brasileiro. 2005;4(3):290-6. doi: 10.1590/ S1677-54492005000300012.

5. Myers K, Wood S, Lee V, Koh P. Variations of connections to the saphenous system in limbs with primary varicose veins: a study in 1481 limbs by duplex ultrasound scanning. J Phlebol. 2002;2:11-7.

6. Hammarsten J, Pedersen P, Cederlund C-G, Campanello $\mathrm{M}$. Long saphenous vein saving surgery for varicose veins. A long-term follow-up. European journal of vascular surgery. 1990;4(4):361-4.

7. Sarquis A. Avaliação pré e pós-operatória no tratamento cirúrgico conservador de varizes tronculares com o duplex scan a cores. Cir Vasc Angiol. 1996;12(4 supl):9-11.

8. Al-Assal F, Gomes A, Al-Assal R, Maffei F, Lastória S, Yoshida W, et al. Tratamento cirúrgico da hipertensão venosa crônica. Maffei FHA, Lastória S, Yoshida WB, Rollo HA Doenças vasculares periféricas 3ª ed São Paulo: Medsi. 2002.

9. Munn S, Morton J, Macbeth W, Mcleish A. To strip or not to strip the long saphenous vein? A varicose veins trial. Br J Surg. 1981;68(6):426-8.

10. Lane R, McMahon C, Cuzzilla M. The treatment of varicose veins using the venous valve cuff. Phlebology. 1994;9(4):136-45. doi: 10.1177/026835559400900402.

11. Zamboni P, Gasbarro V, Marcellino MG, Murgia AP, Feo C, Liboni A, et al. External valvuloplasty of the saphenofemoral junction. Vasc Endovascular Surg. 1994;28(5):327-36. doi: $10.1177 / 153857449402800504$.

12. McMullin G, Smith P, Scurr J. Objective assessment of high ligation without stripping the long saphenous vein. Br J Surg. 1991;78(9):1139-42.

13. Franceschi C. CHIVA effectiveness score: the correct one is below. Eur J Vasc Endovasc Surg. 2012;44(3):351. doi: 10.1016/j.ejvs.2012.06.027.

14. Zamboni P, Cisno C, Marchetti F, Mazza P, Fogato $\mathrm{L}$, Carandina S, et al. Minimally invasive surgical management of primary venous ulcers vs. compression treatment: a randomized clinical trial. Eur J Vasc Endovasc Surg. 2003;25(4):313-8. doi:10.1053/ ejvs.2002.1871.

15. Carandina S, Mari C, De Palma M, Marcellino $\mathrm{M}$, Cisno C, Legnaro A, et al. Varicose vein stripping vs haemodynamic correction (CHIVA): a long term randomised trial. Eur J Vasc Endovasc Surg. 2008;35(2):230-7. doi: 10.1016/j.ejvs.2007.09.011. 
16. Mendoza E, Berger V, Zollmann C, Bomhoff M, Amsler F. Kaliberreduktion der V. saphena magna und der V. femoralis communis nach CHIVA. Phlebologie. 2011;40(2):73-8.

17. Cappelli M, Lova RM, Ermini S, Turchi A, Bono G, Bahnini A, et al. Ambulatory conservative hemodynamic management of varicose veins: critical analysis of results at 3 years. Ann Vasc Surg. 2000;14(4):376-84.

18. Michaels JA, Campbell W, Brazier J, Macintyre J, Palfreyman S, Ratcliffe J, et al. Randomised clinical trial, observational study and assessment of cost-effectiveness of the treatment of varicose veins (REACTIV trial). Health Technol Assess. 2006;10(13):1196, iii-iv.

19. Bellmunt-Montoya S, Escribano JM, Dilme J, Martinez-Zapata MJ. CHIVA method for the treatment of chronic venous insufficiency. Cochrane Database Syst Rev. 2013;(7):CD009648. doi: 10.1002/14651858.CD009648.pub2. 